\title{
Effective Synchronization of a Class of Chua's Chaotic Systems Using an Exponential Feedback Coupling
}

\author{
Patrick Louodop, ${ }^{1}$ Hilaire Fotsin, ${ }^{1}$ Elie B. Megam Ngouonkadi, ${ }^{1}$ \\ Samuel Bowong, ${ }^{2}$ and Hilda A. Cerdeira ${ }^{3}$ \\ ${ }^{1}$ Laboratory of Electronics, Department of Physics, Faculty of Science, University of Dschang, P.O. Box 67, Dschang, Cameroon \\ ${ }^{2}$ Laboratory of Applied Mathematics, Department of Mathematics and Computer Science, Faculty of Science, University of Douala, \\ P.O. Box 24157, Douala, Cameroon \\ ${ }^{3}$ Instituto de Física Teórica-UNESP, Universidade Estadual Paulista, Rua Dr. Bento Teobaldo Ferraz 271, Bloco II, Barra Funda, \\ 01140-070 São Paulo, SP, Brazil
}

Correspondence should be addressed to Hilda A. Cerdeira; cerdeira@ift.unesp.br

Received 17 February 2013; Accepted 4 March 2013

Academic Editor: René Yamapi

Copyright (C) 2013 Patrick Louodop et al. This is an open access article distributed under the Creative Commons Attribution License, which permits unrestricted use, distribution, and reproduction in any medium, provided the original work is properly cited.

A robust exponential function based controller is designed to synchronize effectively a given class of Chua's chaotic systems. The stability of the drive-response systems framework is proved through the Lyapunov stability theory. Computer simulations are given to illustrate and verify the method.

\section{Introduction}

Shortly after Pecora and Carroll showed the possibility of synchronizing chaotic elements [1], applications stretched out in many fields [2-4] giving rise to interdisciplinary research, since this phenomenon appears in many systems in a variety of ways [5-12]. Much research was done developing different strategies in the quest of effective synchronization such as adaptive synchronization [13-15], inverse synchronization [16], and antisynchronization [17-24]. The robustness of many of these methods, as surprising as it may appear, has already been demonstrated in many cases in the presence of noise, perturbations, or parameter mismatches [25-27].

For applications such as telecommunications, where the transmission of messages is not possible unless transmitter and receiver are synchronized [10-12], the investigation of new chaotic systems as well as the most effective means of synchronization is always of great importance. Thus, mathematical models [28, 29], mechanical systems [30], and electronic circuits $[10,13]$ are continually built. One of the best known electronic circuits is the Chua's oscillator [31,32]. Although Chua's circuit is one of the simplest circuits in the literature, it has various complex chaotic dynamics properties which has made it a topic of extensive study [31-34]. A modified version of the circuit has also been topic of attention [33-35]. Its theoretical analysis and numerical simulations agree very well with experimental results.

Recently, some authors proposed a nonlinear controller in order to force synchronization with the purpose of saving energy $[22,23]$. The nonlinear controllers used are based on bounded nonlinear functions $[22,23]$. In this work we apply the exponential function based nonlinear controller to achieve synchronization between the drive-response systems when disturbances are present. Our controller has certain properties which makes it more advantageous to use it, such as the following properties: (1) it is easy to implement in practice; (2) it needs no adaptation algorithm; hence its electrical circuit remains simple; (3) it is faster than the synchronization based on fixed feedback gain which is usually used.

This work is organized as follows. In Section 2, the problem is formulated and the assumptions are given. Section 3 presents the main results. We use Lyapunov stability theory to study the robustness of our proposed controller. We show 


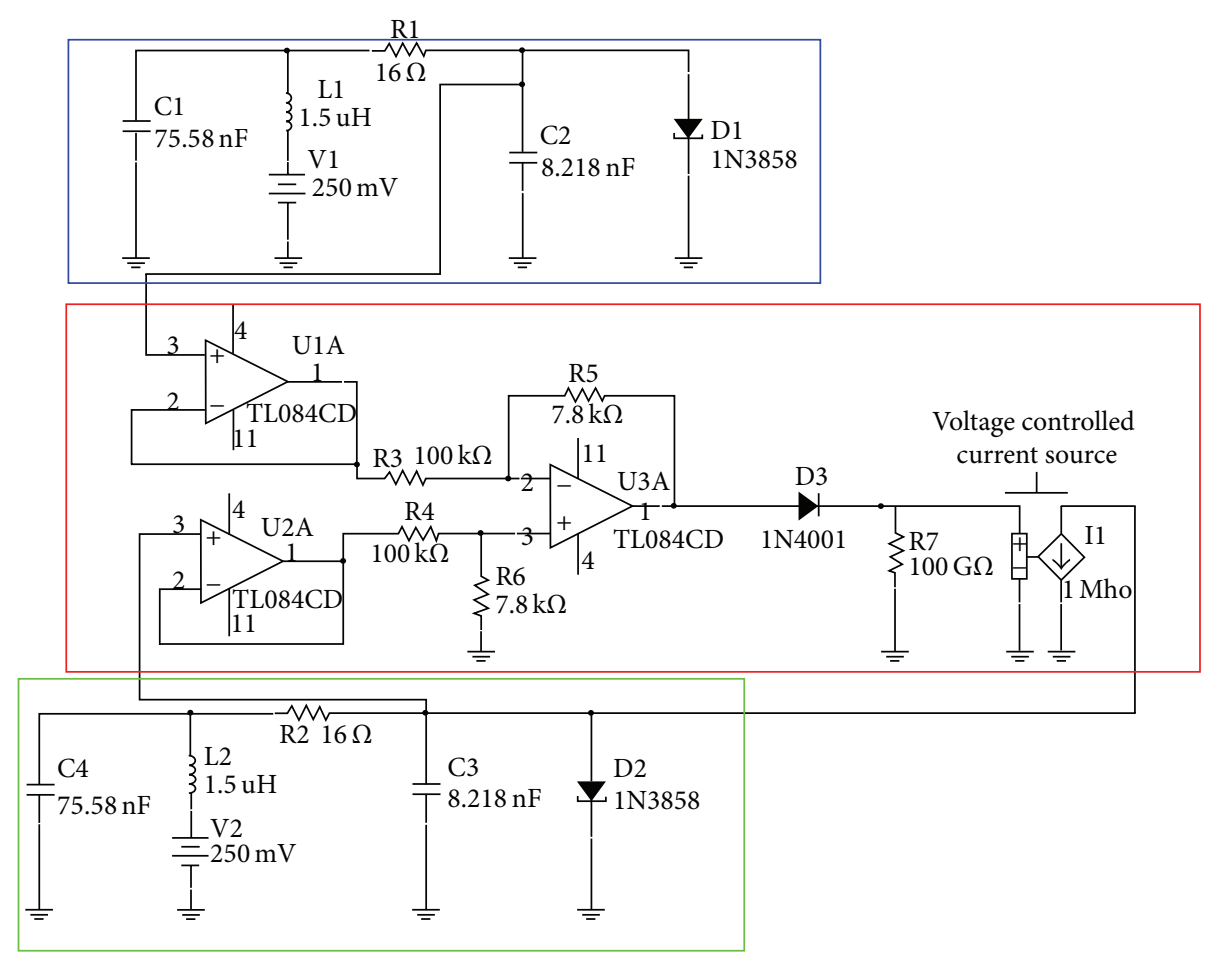

FIGURE 1: Scheme of the circuit for the whole system: transmitter (blue box), controller (red box), and receiver (green box).

that with this controller the drive and response systems are practically synchronized-the errors between the master system and the slave system do not tend to zero but to a limit value. In this case, it is shown that the derivative of Lyapunov function is contained in a closed domain to which the error between master and slave system converges. Since the error is sufficiently small, using the principle of the "ultimate boundedness property," we arrive to the conclusion that the system is globally stable because the derivative of the Lyapunov function is negatively definite. Ultimate boundedness is in particular compatible with local instability about zero and implies global stability. This was demonstrated by Ding and Cheng in [36]. They proposed a new criterion of globally uniformly ultimate boundedness for discrete-time nonlinear systems which helps to relax the condition of stability based on Lyapunov function. The same ideas were successfully applied by de la Sen and Alonso [37], while in [38], Bitsoris et al. work on the robust positive invariance and ultimate boundedness of nonlinear systems with unknown parameters and disturbances, where only their bounds of variance are known. In Section 4, numerical results are presented and we compare the given scheme with that using the simple fixed gain based controller. The conclusions are given in Section 5.

\section{Formulation of the Problem}

In this paper, we study the master-slave synchronization of a class of Chua's chaotic systems, represented in Figure 1 and described by the equations that follow.
The master system is given by:

$$
\begin{gathered}
\dot{x}_{1}(\tau)=\alpha\left[x_{2}(\tau)-x_{1}(\tau)-R f\left(x_{1}(\tau)\right)\right]+d(\tau), \\
\dot{x}_{2}(\tau)=\beta\left[x_{1}(\tau)-x_{2}(\tau)+R x_{3}(\tau)\right], \\
\dot{x}_{3}(\tau)=\gamma\left[v(\tau)-x_{2}(\tau)\right],
\end{gathered}
$$

where $\tau$ is a dimensionless time, $x_{i}(t), i=1,2,3$, are the state variables, $v(\tau)$ is an external force, and $\alpha, \beta, \gamma$, and $R$ are positive constant parameters of the system. The function $f\left(x_{1}(\tau)\right)$ represents the nonlinearity of the system and $d(\tau)$ the disturbances. The function $f\left(x_{1}(\tau)\right)$ defines Chua's circuit, which is given by $f\left(x_{1}\right)=a_{2} x(\tau)+0.5\left(a_{1}-\right.$ $\left.a_{2}\right)\left(\left|x_{1}(\tau)+1\right|-\left|x_{1}(\tau)-1\right|\right)[31,33,34]$, while the modified Chua's system is obtained using $f\left(x_{1}(\tau)\right)=a x_{1}^{3}$ [12] or $f\left(x_{1}(\tau)\right)=a_{1}\left(x_{1}-b\right)^{3}-a_{2}\left(x_{1}-b\right)+a_{3}$ [32]. The latter represents the behavior of a tunnel diode [32]. For an autonomous system $v(\tau)$ is constant and $d(\tau)=0$.

The slave system is given by

$$
\begin{gathered}
\dot{y}_{1}(\tau)=\alpha\left[y_{2}(\tau)-y_{1}(\tau)-R f\left(y_{1}(\tau)\right)\right]+U(\tau), \\
\dot{y}_{2}(\tau)=\beta\left[y_{1}(\tau)-y_{2}(\tau)+R y_{3}(\tau)\right], \\
\dot{y}_{3}(\tau)=\gamma\left[v(\tau)-y_{2}(\tau)\right]
\end{gathered}
$$

where $y_{i}(\tau), i=1,2,3$, is the slave state variables and $U(\tau)$ the feedback coupling.

Here we present a scheme to solve the synchronization problem for system (1). That is to say, if the uncertain system (1) is regarded as the drive system, a suitable response system 
should be constructed to synchronize it with the help of the driving signal $x$. In order to do so, we assume the following:

(i) There is a bounded region $\mathscr{U} \subset R^{3}$ containing the whole basin of the drive system (1) such that no orbit of system (1) ever leaves it.

(ii) The disturbance $d(\tau)$ is bounded by an unknown positive constant $D$, namely,

$$
\|d(\tau)\| \leq D,
$$

where $\|\cdot\|$ denotes the euclidian norm of a vector.

(iii) All chaotic systems are supposed to be confined to a limited domain; hence there exists a positive constant $L$ such that

$$
\left\|f\left(y_{1}(\tau)\right)-f\left(x_{1}(\tau)\right)\right\| \leq L\left\|y_{1}(\tau)-x_{1}(\tau)\right\| .
$$

We will now try to synchronize the systems described in (1) and (2) designing an appropriate control $U(\tau)$ in system (2) such that

$$
\left\|y_{i}(\tau)-x_{i}(\tau)\right\| \leq r, \quad \text { for } \tau \longrightarrow \infty,
$$

where $r$ is a sufficiently small positive constant.

Let us define the state errors between the transmitter and the receiver systems as

$$
e_{i}(\tau)=y_{i}(\tau)-x_{i}(\tau), \quad \text { with } i=1,2,3,
$$

and the feedback coupling as

$$
U(\tau)=-\varphi\left(\exp \left(k e_{1}(\tau)\right)-1\right),
$$

where $\varphi$ and $k$ are positive fixed constants.

Introducing the definition of the systems (1), (2), and (7) into (2), the dynamics of the error states (6) becomes

$$
\begin{gathered}
\dot{e}_{1}(\tau)=\alpha\left[e_{2}(\tau)-e_{1}(\tau)-R\left(f\left(y_{1}\right)-f\left(x_{1}\right)\right)\right] \\
-d(\tau)-\varphi\left(\exp \left(k e_{1}\right)-1\right) \\
\dot{e}_{2}(\tau)=\beta\left[e_{1}(\tau)-e_{2}(\tau)+R e_{3}(\tau)\right] \\
\dot{e}_{3}(\tau)=-\gamma e_{2}(\tau)
\end{gathered}
$$

The problem is now reduced to demonstrating that with the chosen control law $U(\tau)$, the error states $e_{i}, i=1,2,3$, in (8) are at most a sufficiently small positive constant $r$, which will prove the proposition.

\section{Main Results}

If we consider the master-slave chaotic systems (1) and (2) with all the aforementioned assumptions (3) and with the exponential function based feedback coupling given by the relation (7), we will show that the overall system will be practically synchronized, that is, $\left\|y_{i}(\tau)-x_{i}(\tau)\right\| \leq r$, where $r$ is a sufficiently small positive constant for large enough $\tau$.

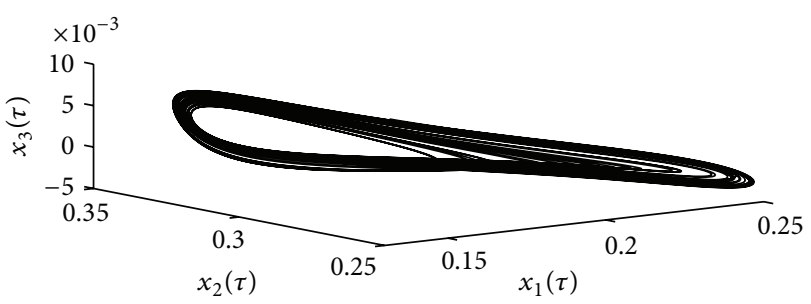

FIgURE 2: 3D chaotic attractor of the tunnel diode based modified Chua's system.

In order to do so, let us consider the following Lyapunov function:

$$
V=\frac{1}{2}\left[\frac{e_{1}^{2}}{\alpha}+\frac{e_{2}^{2}}{\beta}+\frac{R e_{3}^{2}}{\gamma}\right] .
$$

Differentiating the function $V$ with respect to time yields

$$
\begin{aligned}
\dot{V}= & 2 e_{1} e_{2}-e_{1}^{2}-e_{2}^{2}-R\left(f\left(y_{1}\right)-f\left(x_{1}\right)\right) e_{1} \\
& -\frac{d(\tau)}{\alpha} e_{1}-\frac{\varphi}{\alpha}\left[\exp \left(k e_{1}\right)-1\right] e_{1} \\
= & -\left(e_{1}-e_{2}\right)^{2}-R\left(f\left(y_{1}\right)-f\left(x_{1}\right)\right) e_{1} \\
& -\frac{d(\tau)}{\alpha} e_{1}-\frac{\varphi}{\alpha}\left[\exp \left(k e_{1}\right)-1\right] e_{1} .
\end{aligned}
$$

Expanding the exponential function as follows:

$$
\begin{aligned}
& \exp \left(k e_{1}\right)-1 \simeq k e_{1}+\sum_{i=1}^{n} \frac{\left(k e_{1}\right)^{2 i}}{2 i !}+\sum_{i=1}^{n} \frac{\left(k e_{1}\right)^{2 i+1}}{(2 i+1) !} \\
&+\theta\left(e_{1}\right)+\zeta\left(e_{1}\right),
\end{aligned}
$$

where $\theta\left(e_{1}\right)$ and $\zeta\left(e_{1}\right)$ constitute the rest of the expansion in order greater than $n$ for odd part and for even part of the development, respectively, and substituting by the maximum value of the disturbance, $D$, it follows that

$$
\begin{aligned}
\dot{V} \leq & R L e_{1}^{2}+\frac{D}{\alpha}\left|e_{1}\right| \\
& -\frac{\varphi}{\alpha}\left(k e_{1}+\sum_{i=1}^{n} \frac{\left(k e_{1}\right)^{2 i}}{2 i !}+\theta\left(e_{1}\right)\right) e_{1} .
\end{aligned}
$$

Hence, we have

$$
\begin{aligned}
\dot{V} \leq & \left(R L-\frac{\varphi}{\alpha} k\right) e_{1}^{2} \\
& +\frac{1}{\alpha}\left(D+\varphi \sum_{i=1}^{n} \frac{\left(k\left|e_{1}\right|\right)^{2 i}}{2 i !}+\left|\theta\left(e_{1}\right)\right|\right)\left|e_{1}\right|, \\
\dot{V} \leq & \left(R L-\frac{\varphi}{\alpha} k\right) e_{1}^{2} \\
& +\frac{1}{\alpha}\left(D+\varphi \sum_{i=1}^{n} \frac{(k r)^{2 i}}{(2 i) !}+\Theta(r)\right) r,
\end{aligned}
$$

where $\Theta(r) \geq \max \left(\theta\left(e_{1}\right)\right)$. 


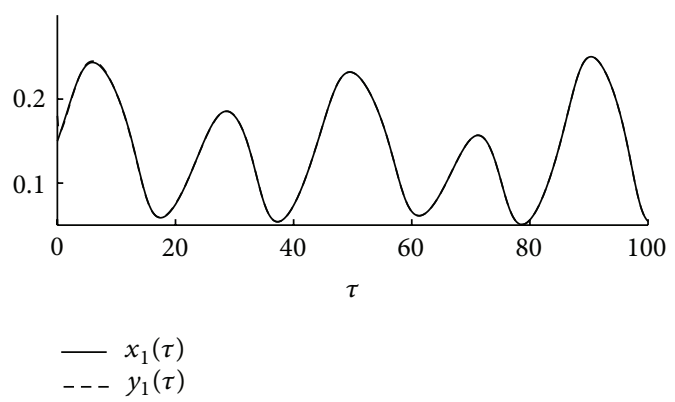

(a)

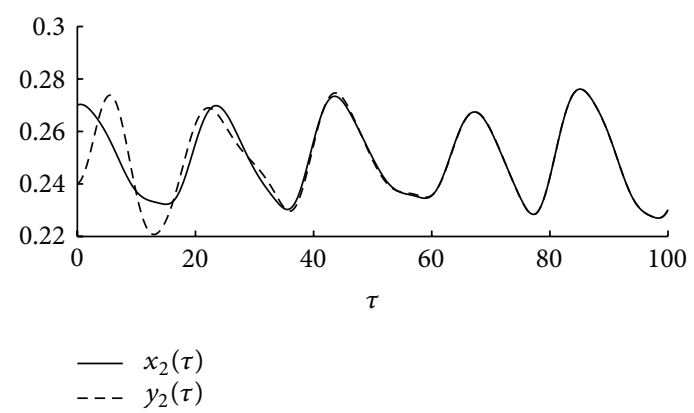

(c)

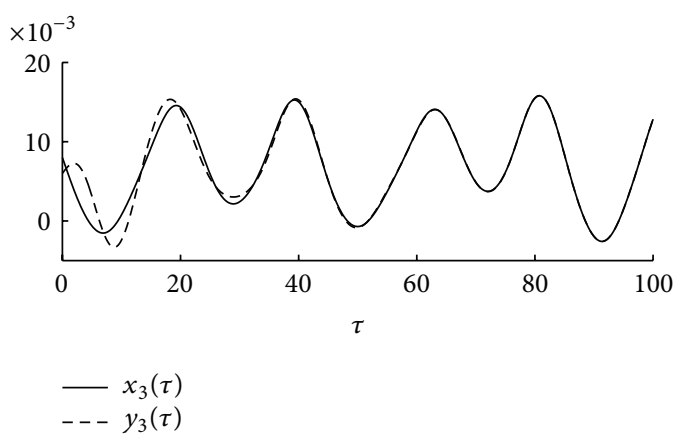

(e)

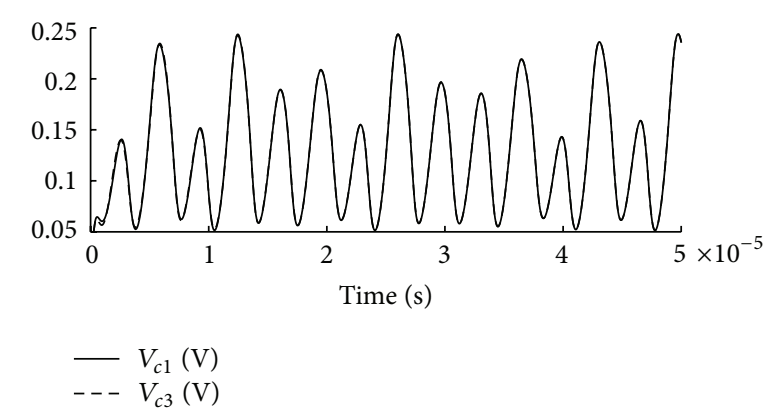

(b)

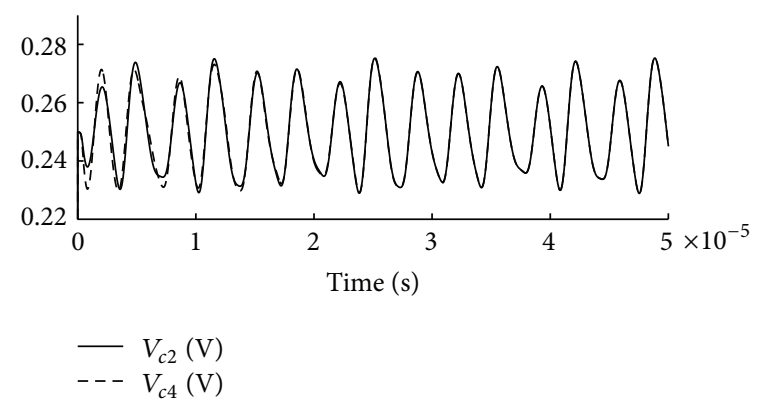

(d)

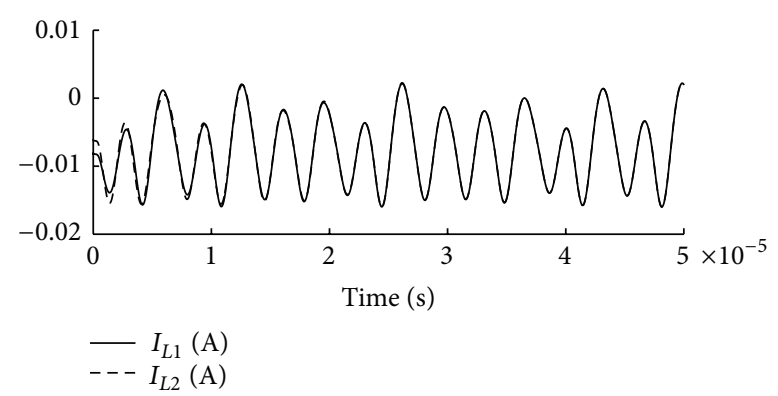

(f)

FIgURE 3: Time evolution of the master system (solid lines) and slave system (dashed lines) from Matlab simulations (left) and Pspice simulations (right).

Here we use $r$ as an upper bound for the error in each axis. Then we see that the derivative of the Lyapunov function (12) is lower than that in (13), which in turn is smaller than the one given by (14). Thus expression (14) is maximized and the radius of the close domain to which the error is attracted is determined. Defining

$$
\varphi \geq \frac{\alpha R L}{k}, \quad \phi=\left(\frac{D+\Theta(r)}{\alpha}+\frac{R L}{k} \sum_{i=1}^{n} \frac{(k r)^{2 i}}{(2 i) !}\right) r
$$

one obtains

$$
\dot{V} \leq-\psi e_{1}^{2}+\phi, \quad \text { where } \psi=\left|R L-\frac{\varphi}{\alpha} k\right| .
$$

Equation (16) is in principle a form of the ultimate boundedness property in the sense that if the error is sufficiently small, then the system is globally stable because the upperbound is negative [36]. From (16), it follows that if

$$
\left\|e_{1}\right\|>\sqrt{\frac{\phi}{\psi}}
$$

therefore, $\dot{V}(\tau)<0$; hence $V(\tau)$ decreases, which implies that $\left\|e_{1}\right\|$ decreases. It then follows from standard invariance arguments as in [23] that asymptotically for increasing time the error satisfies the following bound

$$
\left\|e_{1}\right\|<C
$$

for any $C>\sqrt{\phi / \psi}$.

So if $\phi$ is sufficiently small, the bound for the synchronization error will also be sufficiently small. Therefore, the synchronization state error would be contained within a neighborhood of the origin, as we wanted to prove. In 


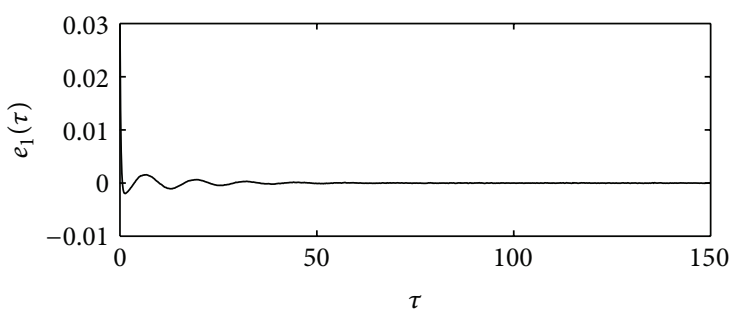

(a)

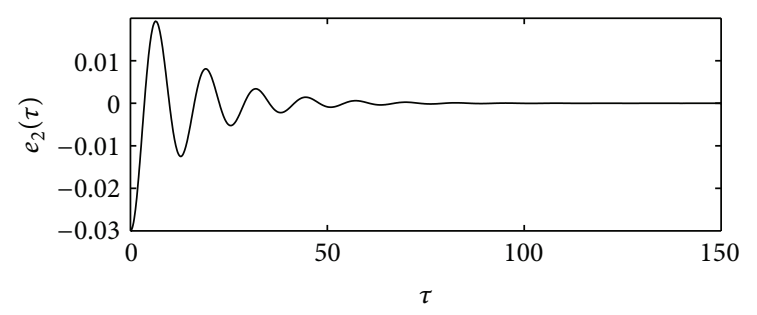

(c)

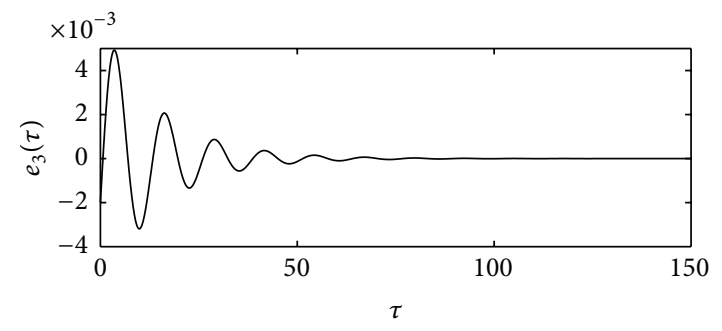

(e)

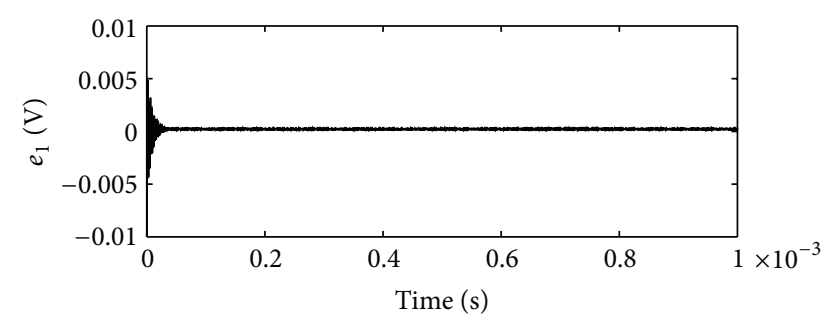

(b)

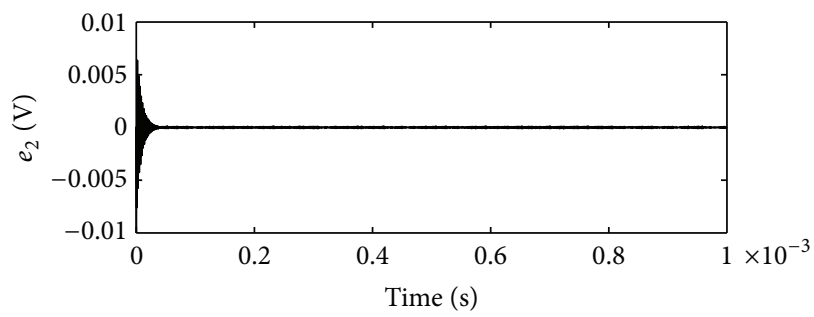

(d)

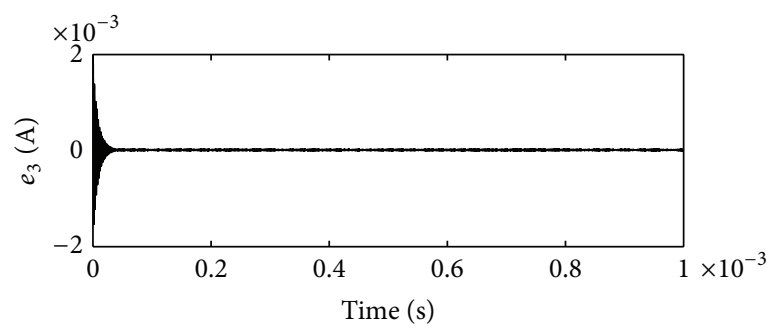

(f)

Figure 4: Time evolution of the synchronization errors from Matlab simulations (left) and Pspice simulations (right).

addition as $V(\tau)$ decreases, then there exists a continuous and strictly increasing function $\varrho$ and a finite integer $\eta$, such that

$$
V(e(\tau+\eta), \tau+\eta)-V(e(\tau), \tau) \leq-\varrho(\|e(\tau)\|),
$$

where $e(\tau)=\left(e_{1}(\tau), e_{2}(\tau), e_{3}(\tau)\right)$.

Thus, the Lyapunov function respects [36, Theorem 3.1] and then (8) is globally uniformly ultimate bounded near the origin.

\section{Numerical Simulations}

4.1. Chaotic Systems. In this section, we present some numerical results for the circuit shown in Figure 1, to illustrate the effectiveness of the proposed scheme, where the threedimensional tunnel diode based modified Chua's system [35] is used as transmitter (blue box) and receiver (green box), the controller appears inside the red box. With the initial conditions selected as $\left(x_{1}(0), x_{2}(0), x_{3}(0)\right)=(0.15,0.27,0.008)$ and $\left(y_{1}(0), y_{2}(0), y_{3}(0)\right)=(0.18,0.24,0.006)$ and with the given system's parameters: $\alpha=2.507463, \beta=0.2985075$, $\gamma=0.20875, R=16, e=0.250, a_{1}=1.3242872, a_{2}=$ $0.06922314, a_{3}=0.00539$, and $b=0.167$, the systems behave chaotically as shown in Figure 2. The disturbances $d(\tau)$ are given by the relation $d(\tau)=0.001 \mathrm{wgn}(1,1,1)\left(x_{1}(\tau)+x_{2}(\tau)\right)$, where $\operatorname{wgn}(1,1,1)$ is Matlab white gaussian noise generator.
4.2. Simulation Results and Discussion. The controller's parameters are $\varphi=10$ and $k=3$. The controller circuit was realized through the following relations: $k=R_{5} / V_{T} R_{3}=$ $R_{6} / V_{T} R_{4}$ and $\varphi=R_{7} I_{s}$ where $V_{T} \simeq 0.026$ Volt and $I_{s} \simeq 10^{-12}$ are diode characteristics. The Voltage controlled current source (VCCS) is used to minimize as much as possible the mutual influence of between the slave system (Green box) and the controller (Red box) and to only generate the current which obliges the response system to follow the drive system. The graphs of Figures 3 and 4 show that the synchronization is reached around the dimensionless time $\tau=60$.

Remark 1. In Pspice simulations, the synchronization is reached for high values of $R_{7}$ particularly if $R_{7}>100 \mathrm{k} \Omega$. $R_{7}$ role is to increase the value of the VCCS output current by increasing the value of the voltage at its landmarks.

Considering the case without disturbances, if we compare the proposed scheme with the one for which the controller is given by the following relation:

$$
U(t)=-\zeta e_{1}(\tau),
$$

where $\zeta$ is a positive constant chosen equal to $\varphi$, it appears that, as one can visually appreciate on the graphs of Figures 5 and 6 , the exponential function based nonlinear controller is faster than the linear controller with fixed gain. 


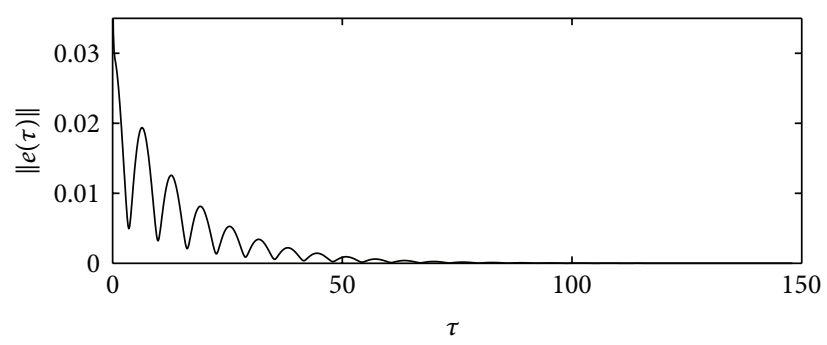

(a)

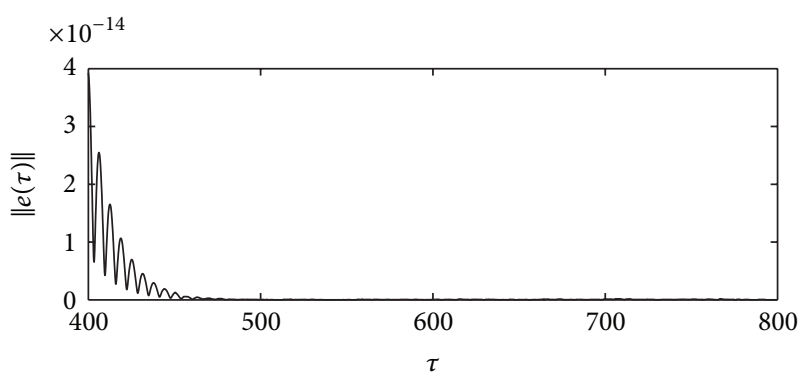

(b)

FIGURE 5: Time evolution of the synchronization errors norm with the propose scheme (7).

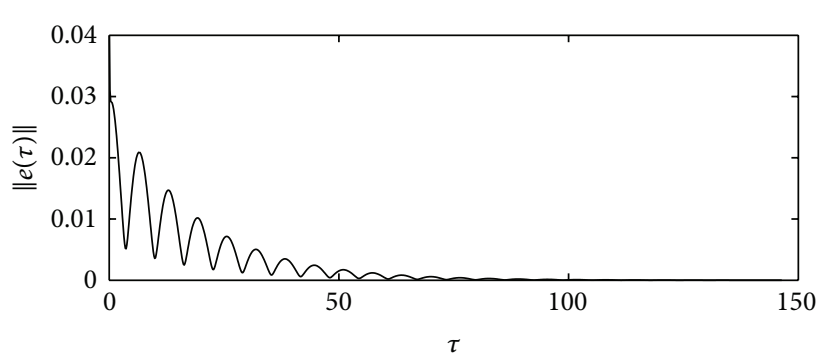

(a)

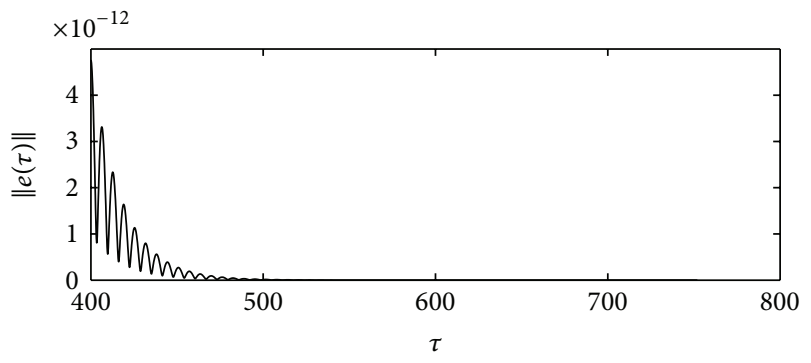

(b)

FIgURE 6: Time evolution of the synchronization errors norm with linear controller (20).

\section{Conclusion}

In this paper the synchronization between two different delayed chaotic systems is studied via a simple-exponential function based-nonlinear controller. Although different initial conditions and disturbances make synchronization more difficult, a simple exponential function based nonlinear controller is designed which facilitates the task. This is proven through the Lyapunov stability theory; it is shown that both master-slave systems should be practically synchronized. It is important to note that the proposed scheme improves the linear controller with fixed gain usually used. To show the effectiveness of the proposed strategy, some numerical simulations are given; they show the efficiency of the proposed strategy in front of the linear fixed gain based controller. The electronic circuit of the used controller is also given followed by some simulations.

\section{Acknowledgments}

The authors thank the hospitality of the University of Yaounde I and the Abdus Salam International Centre for Theoretical Physics. H. A. Cerdeira acknowledges support by the CNPq-ProAfrica, project no. 490265/2010-3 (Brazil). P. Louodop acknowledges M. André Romet for support throughout this work.

\section{References}

[1] L. M. Pecora and T. L. Carroll, "Synchronization in chaotic systems," Physical Review Letters, vol. 64, no. 8, pp. 821-824, 1990.
[2] X. Li, X. Guan, and D. Ru, "The damping time of EEG with information retrieve and autoregressive models," in Proceedings of the 5th IFAC Symposium on Modelling and Control in Biomedical Systems, Melbourne, Australia, August 2003.

[3] S. K. Han, C. Kurrer, and Y. Kuramoto, "Dephasing and bursting in coupled neural oscillators," Physical Review Letters, vol. 75, no. 17, pp. 3190-3193, 1995.

[4] J. S. Lin, C. F. Huang, T. L. Liao, and J. J. Yan, "Design and implementation of digital secure communication based on synchronized chaotic systems," Digital Signal Processing, vol. 20, no. 1, pp. 229-237, 2010.

[5] N. Islam, B. Islam, and H. P. Mazumdar, "Generalized chaos synchronization of unidirectionally coupled Shimizu-Morioka dynamical systems," Differential Geometry, vol. 13, pp. 101-106, 2011.

[6] B. Blasius, A. Huppert, and L. Stone, "Complex dynamics and phase synchronization in spatially extended ecological systems," Nature, vol. 399, no. 6734, pp. 354-359, 1999.

[7] S. Sivaprakasam, I. Pierce, P. Rees, P. S. Spencer, K. A. Shore, and A. Valle, "Inverse synchronization in semiconductor laser diodes," Physical Review A, vol. 64, no. 1, pp. 138051-138058, 2001.

[8] I. Wedekind and U. Parlitz, "Synchronization and antisynchronization of chaotic power drop-outs and jump-ups of coupled semiconductor lasers," Physical Review E, vol. 66, no. 2, Article ID 026218, pp. 1-4, 2002.

[9] A. Fradkov, H. Nijmeijer, and A. Markov, "Adaptive observerbased synchronization for communication," International Journal of Bifurcation and Chaos in Applied Sciences and Engineering, vol. 10, no. 12, pp. 2807-2813, 2000.

[10] K. M. Cuomo and A. V. Oppenheim, "Circuit implementation of synchronized chaos with applications to communications," Physical Review Letters, vol. 71, no. 1, pp. 65-68, 1993. 
[11] S. Bowong, "Stability analysis for the synchronization of chaotic systems with different order: application to secure communications," Physics Letters A, vol. 326, no. 1-2, pp. 102-113, 2004.

[12] S. Bowong and J. J. Tewa, "Unknown inputs' adaptive observer for a class of chaotic systems with uncertainties," Mathematical and Computer Modelling, vol. 48, no. 11-12, pp. 1826-1839, 2008.

[13] H. Fotsin and S. Bowong, "Adaptive control and synchronization of chaotic systems consisting of Van der Pol oscillators coupled to linear oscillators," Chaos, Solitons and Fractals, vol. 27, no. 3, pp. 822-835, 2006.

[14] A. Astolfi, D. Karagiannis, and R. Ortega, Nonlinear and Adaptive Control with Applications, Springer, London, UK, 2008.

[15] G. Feng and R. Lozano, Adaptive Control Systems, Reed Elsevier, 1999.

[16] E. M. Shahverdiev, R. A. Nuriev, L. H. Hashimova, E. M. Huseynova, R. H. Hashimov, and K. A. Shore, "Complete inverse chaos synchronization, parameter mismatches and generalized synchronization in the multi-feedback Ikeda model," Chaos, Solitons and Fractals, vol. 36, no. 2, pp. 211-216, 2008.

[17] V. Sundarapandian, "Global chaos anti-synchronization of Liu and Chen systems by nonlinear control," International Journal of Mathematical Sciences \& Applications, vol. 1, no. 2, pp. 691-702, 2011.

[18] X. Zhang and H. Zhu, "Anti-synchronization of two different hyperchaotic systems via active and adaptive control," International Journal of Nonlinear Science, vol. 6, no. 3, pp. 216-223, 2008.

[19] H. Zhu, "Anti-synchronization of two different chaotic systems via optimal control with fully unknown parameters," Information and Computing Science, vol. 5, pp. 11-18, 2010.

[20] X. Gao, S. Zhong, and F. Gao, "Exponential synchronization of neural networks with time-varying delays," Nonlinear Analysis. Theory, Methods \& Applications A, vol. 71, no. 5-6, pp. 20032011, 2009.

[21] S. Zheng, Q. Bi, and G. Cai, "Adaptive projective synchronization in complex networks with time-varying coupling delay," Physics Letters A, vol. 373, no. 17, pp. 1553-1559, 2009.

[22] J. Cai, M. Lin, and Z. Yuan, "Secure communication using practical synchronization between two different chaotic systems with uncertainties," Mathematical \& Computational Applications, vol. 15, no. 2, pp. 166-175, 2010.

[23] P. Louodop, H. Fotsin, and S. Bowong, "A strategy for adaptive synchronization of an electrical chaotic circuit based on nonlinear control," Physica Scripta, vol. 85, no. 2, Article ID 025002, 2012.

[24] M. Roopaei and A. Argha, "Novel adaptive sliding mode synchronization in a class of chaotic systems," World Applied Sciences Journal, vol. 12, pp. 2210-2217, 2011.

[25] Z. Sun and X. Yang, "Parameters identification and synchronization of chaotic delayed systems containing uncertainties and time-varying delay," Mathematical Problems in Engineering, vol. 2010, Article ID 105309, 15 pages, 2010.

[26] S. T. Kammogne and H. B. Fotsin, "Synchronization of modified Colpitts oscillators with structural perturbations," Physica Scripta, vol. 83, no. 6, Article ID 065011, 2011.

[27] C. K. Ahn, "Robust chaos synchronization using input-to-state stable control," Pramana, vol. 74, no. 5, pp. 705-718, 2010.

[28] D. J. D. Earn, P. Rohani, and B. T. Grenfell, "Persistence, chaos and synchrony in ecology and epidemiology," Proceedings of the Royal Society B, vol. 265, no. 1390, pp. 7-10, 1998.
[29] S. Bowong, "Optimal control of the transmission dynamics of tuberculosis," Nonlinear Dynamics, vol. 61, no. 4, pp. 729-748, 2010.

[30] Z. Yang, G. K. M. Pedersen, and J. H. Pedersen, "Model-based control of a nonlinear one dimensional magnetic levitation with a permanent-magnet object," in Automation and Robotics, chapter 21, pp. 359-374.

[31] L. O. Chua, "The genesis of Chua's circuit," AEU. Archiv fur Elektronik und Ubertragungstechnik, vol. 46, no. 4, pp. 250-257, 1992.

[32] L. O. Chua, T. Yang, G. Q. Zhong, and C. W. Wu, "Synchronization of Chua's circuits with tune-varying channels and parameters," IEEE Transactions on Circuits and Systems I, vol. 43, no. 10, pp. 862-868, 1996.

[33] Y.Z. Yin, "Experimental demonstration of chaotic synchronization in the modified Chua's oscillators," International Journal of Bifurcation and Chaos in Applied Sciences and Engineering, vol. 7, no. 6, pp. 1401-1410, 1997.

[34] X. X. Liao, H. G. Luo, G. Zhang, J. G. Jian, X. J. Zong, and B. J. Xu, "New results on global synchronization of Chua's circuit," Acta Automatica Sinica, vol. 31, no. 2, pp. 320-326, 2005.

[35] A. Y. Markov, A. L. Fradkov, and G. S. Simin, "Adaptive synchronization of chaotic generators based on tunnel diodes," in Proceedings of the 35th IEEE Conference on Decision and Control, pp. 2177-2182, December 1996.

[36] Z. Ding and G. Cheng, "A new uniformly ultimate boundedness criterion for discrete-time nonlinear systems," Applied Mathematics, vol. 2, pp. 1323-1326, 2011.

[37] M. de la Sen and S. Alonso, "Adaptive control of time-invariant systems with discrete delays subject to multiestimation," Discrete Dynamics in Nature and Society, vol. 2006, Article ID 41973, 27 pages, 2006.

[38] G. Bitsoris, M. Vassilaki, and N. Athanasopoulos, "Robust positive invariance and ultimate bounded- ness of nonlinear systems," in Proceedings of the 20th Mediterranean Conference on Control and Automation (MED), pp. 598-603, Barcelona, Spain, July 2012. 


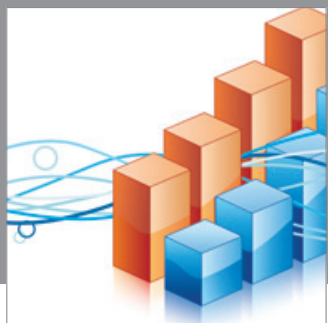

Advances in

Operations Research

mansans

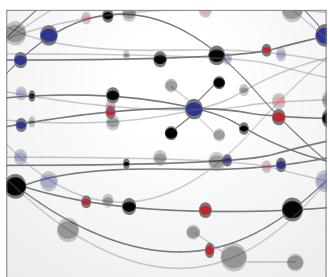

The Scientific World Journal
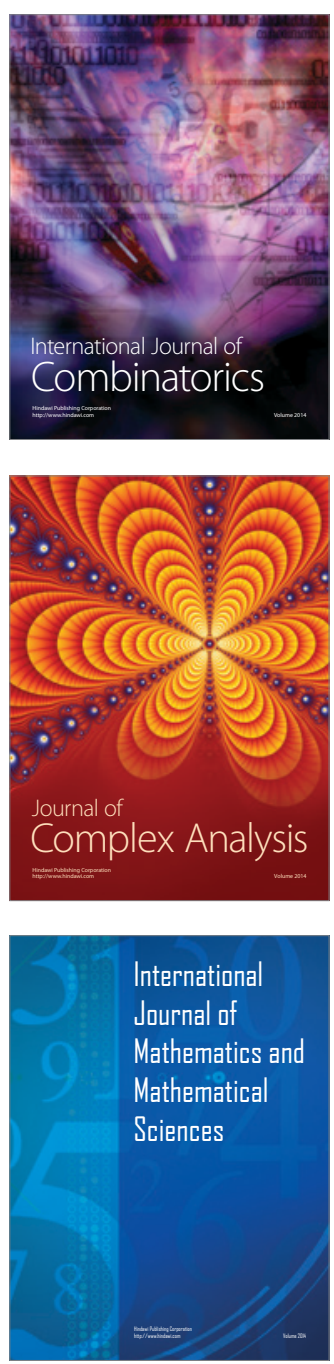
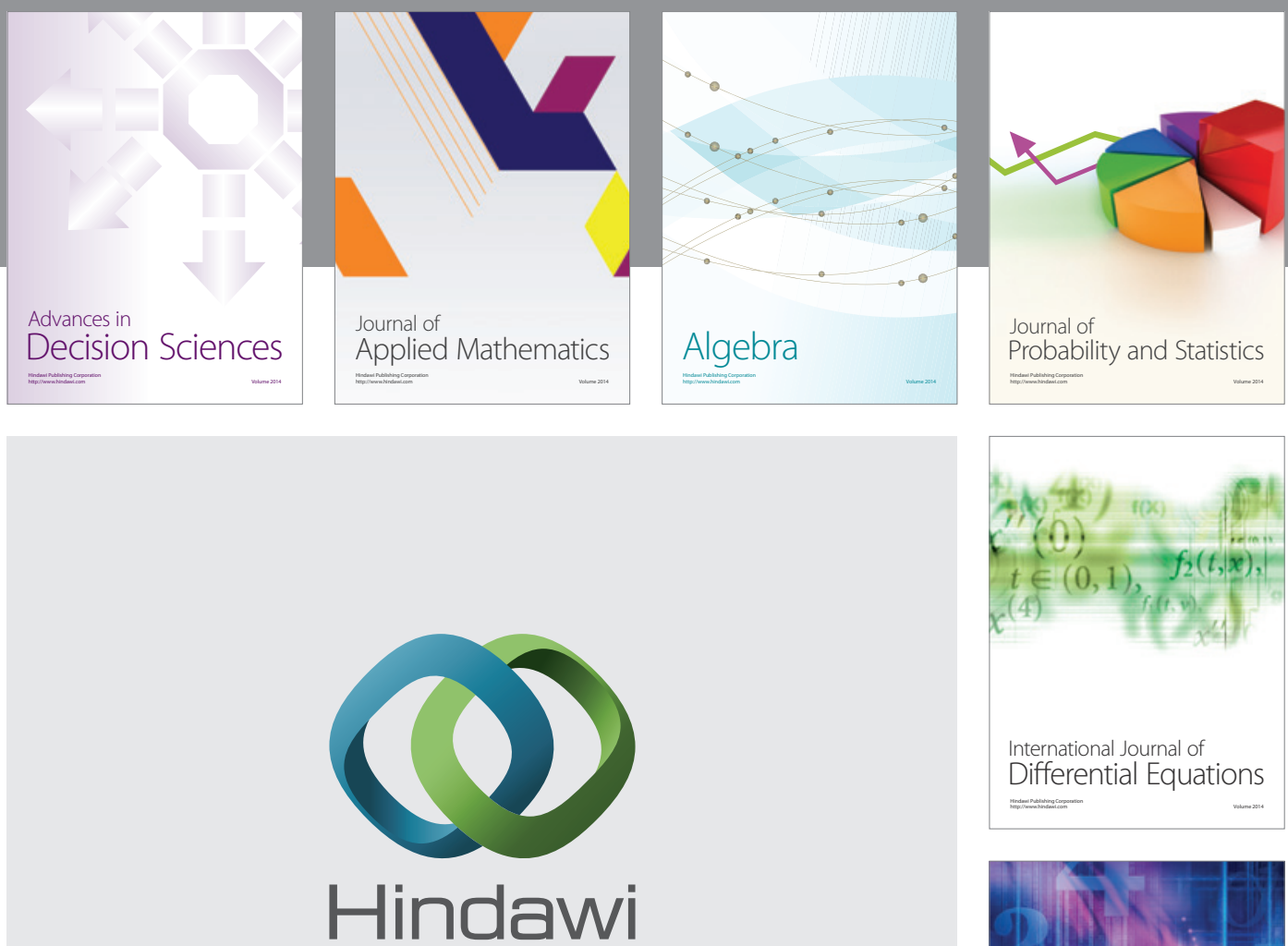

Submit your manuscripts at http://www.hindawi.com
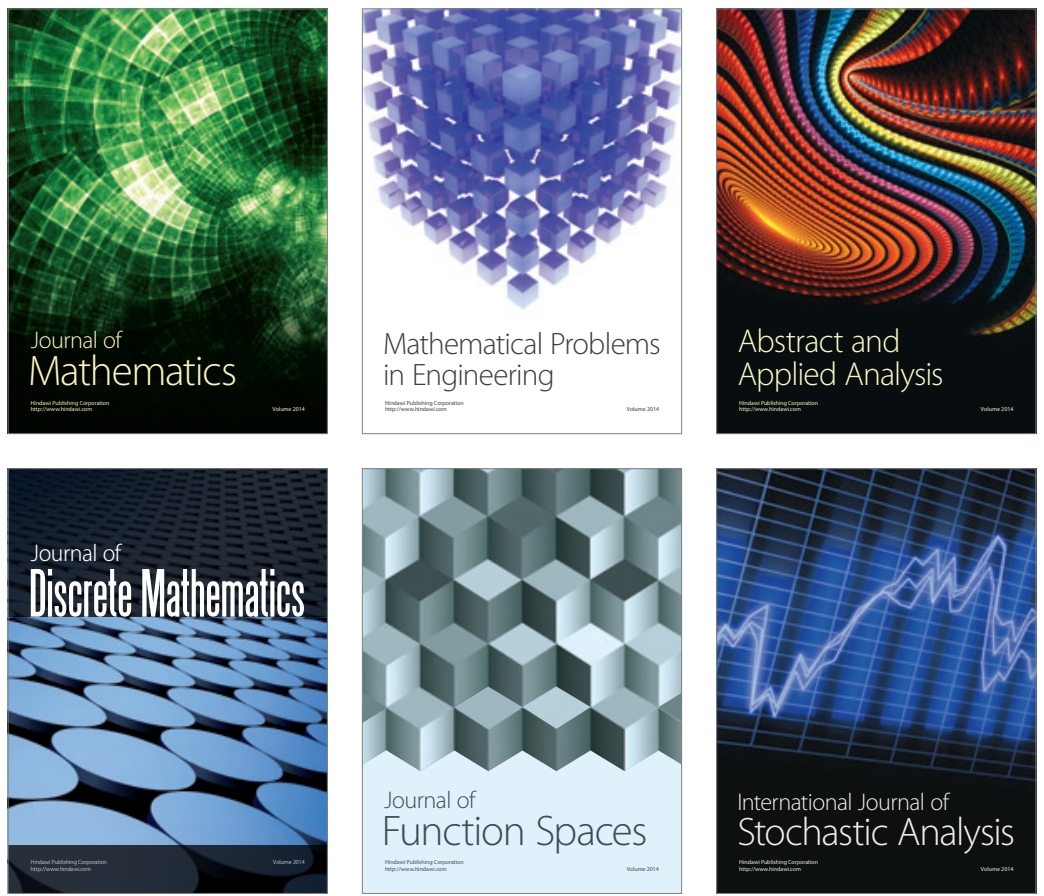

Journal of

Function Spaces

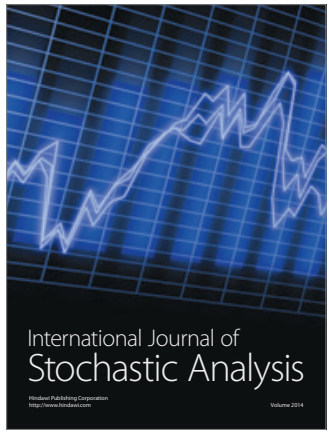

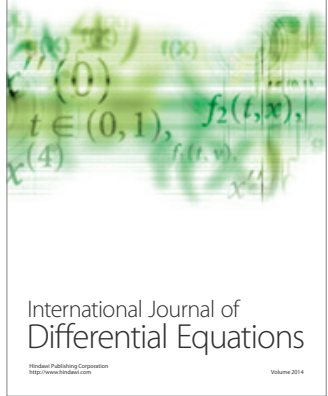
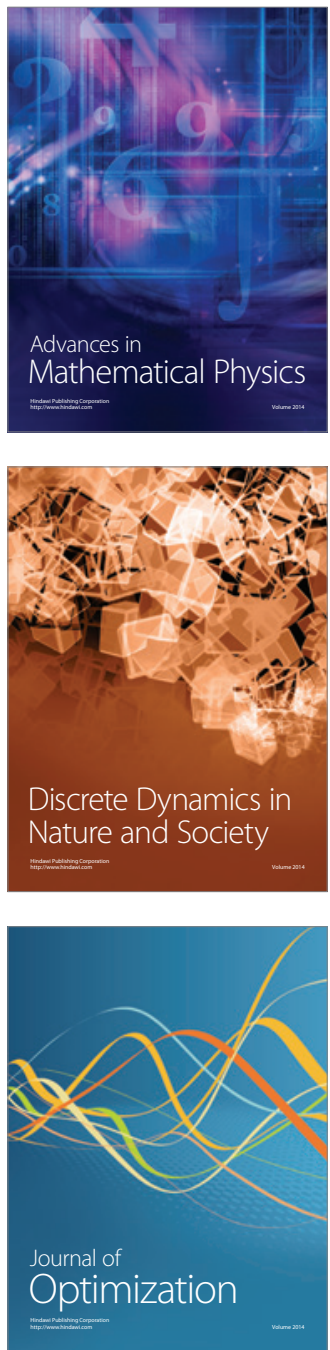